Published in final edited form as:

Lancet. 2005 August 13; 366(9485): 530-531.

\title{
Risk stratification in Brugada syndrome
}

\author{
Kathryn A Glatter ${ }^{*}$, Nipavan Chiamvimonvat, Matti Viitasalo, Qing Wang, and Dipika Tuteja \\ Department of Cardiology, University of California, Davis, Sacramento, CA 95817, USA (KAG, NC, \\ DT); Department of Cardiology, Helsinki University Hospital, Helsinki, Finland (MV); and Center for \\ Cardiovascular Genetics and Department of Molecular Cardiology Cardiology, Cleveland Clinic \\ Foundation, Cleveland, Ohio, USA (QW)
}

It is easy to recommend an implantable cardioverter-defibrillator to a patient who has just survived a cardiac arrest, but what do you tell his asymptomatic brother who has the same genetic disease? In this age of gene testing and sophisticated technology, such questions arise frequently, and the answers are complex.

Lars Eckardt and colleagues recently addressed this question of risk stratification for asymptomatic patients with Brugada syndrome. ${ }^{1}$ Brugada syndrome is a genetic disease characterised by mutations in the cardiac sodium-channel (SCN5A), which cause a loss of function of the channel. ${ }^{2-4}$ Patients with Brugada syndrome have normal heart function but are prone to cardiac arrhythmias and sudden death, especially in middle-aged men. The syndrome is one of the leading causes of death for young men in south-east Asia, where the mutation is particularly common. 5

Like most such genetic causes of sudden death, Brugada syndrome is an autosomal dominant disease, meaning that $50 \%$ of the progeny will inherit the mutation (and risk of sudden death) from the affected proband. Thus there will probably be more silent carriers of the mutation who may never exhibit disease symptoms (syncope, cardiac arrest, or sudden death) than symptomatic probands. Although drugs such as quinidine and sotalol have been tried in patients with Brugada syndrome, the only real treatment is placement of an implantable cardioverterdefibrillator to prevent sudden death. 6,7 Diagnosis of these patients is also problematic because the characteristic ECG findings (figure) may be absent. Genetic testing will only reveal the mutation in $20 \%$ of patients with Brugada syndrome. How to treat these asymptomatic patients is controversial. ${ }^{8}$

Two major research groups have studied Brugada syndrome and provide much of the available data. The first group is an international registry spearheaded by the Brugada brothers, who initially described the disease in $1992 .{ }^{9}$ Their cohort of patients is the largest but appears to be one at relatively high-risk for sudden death. Their group reported the clinical prognosis of 547 patients with Brugada syndrome over 24 (SD 33) months of follow-up. ${ }^{2}$ Despite the short follow-up, $8 \%$ of the initially asymptomatic patients died or had ventricular fibrillation. The strongest predictor of adverse outcome was a positive invasive electrophysiology study during which malignant ventricular arrhythmias were induced. Inducible individuals had a six-fold increased risk of sudden death or ventricular fibrillation during the subsequent 2 years compared with non-inducible subjects.

The second research group led by Priori et al in Italy reported the natural history of 200 patients with Brugada syndrome. ${ }^{10}$ Inducibility at electrophysiology study was not predictive of

\footnotetext{
*kaglatter@ucdavis.edu.

Portions of our research work are supported by a Beginning-Grant-in-Aid, Western States Affiliates, and the Pfizer/SWHR Foundation to KAG. Otherwise, we declare that we have no conflict of interest.
} 
untoward clinical outcomes in their cohort. The highest risk feature was an ECG consistent with the diagnosis at baseline and a history of syncope; $44 \%$ of these patients had a cardiac arrest. Their group advocated placement of an implantable cardioverter-defibrillator in such patients and no treatment in asymptomatic patients whose baseline ECG was normal.

Eckardt and colleagues describe a large cohort with Brugada syndrome (212 patients) from four European centres with the longest follow-up yet published (40 [SD 50] months). Only $1 \%$ of the initially asymptomatic patients had an arrhythmic episode during follow-up. Their cohort seemed to be at lower risk for sudden death than the Brugada registry, perhaps because of a selection bias in the latter group. Importantly, Eckardt did not find that electrophysiology study was useful for risk stratification. Indeed, such testing was negative in four of nine Brugada syndrome patients who eventually had clinical events. Their sentinel work is highly useful because they show that asymptomatic patients with incidentally discovered ECGs consistent with Brugada syndrome are at relatively low-risk for cardiac events.

The Second Consensus Conference on Brugada syndrome recently recommended placement of an implantable cardioverter-defibrillator for cardiac-arrest survivors but admitted that it is unclear if asymptomatic patients should receive treatment. ${ }^{11}$ Eckardt and colleagues' study suggests that such patients will do well and do not require intervention. Additionally, they did not find that invasive electrophysiology study was a useful tool for risk-stratification and should not be used.

All groups sound a word of caution with their findings due to the short follow-up in their studies. Whether initially asymptomatic patients could have a cardiac arrest decades after their diagnosis is unknown. Such findings will only be revealed over time. Affected family members who have not yet had symptoms must wait as we all continue to learn more about the natural history of Brugada syndrome.

\section{References}

1. Eckardt L, Probst V, Smits JPP, et al. Long-term prognosis of individuals with right precordial STsegment-elevation Brugada syndrome. Circulation 2005;111:257-63. [PubMed: 15642768]

2. Brugada J, Brugada R, Brugada P. Determinants of sudden cardiac death in individuals with the electrocardiographic pattern of Brugada syndrome and no previous cardiac arrest. Circulation 2003;108:3092-96. [PubMed: 14623800]

3. Wang Q, Li Z, Shen J, et al. Genomic organization of the human SCN5A gene encoding the cardiac sodium channel. Genomics 1996;34:9-16. [PubMed: 8661019]

4. Chen Q, Kirsch GE, Zhang D, et al. Genetic basis and molecular mechanism for idiopathic ventricular fibrillation. Nature 1998;392:293-96. [PubMed: 9521325]

5. Veerakul G, Nademanee K. What is the sudden death syndrome in Southeast Asian males? Cardiol Rev 2000;8:90-95. [PubMed: 11174879]

6. Belhassen B, Glick A, Viskin S. Efficacy of quinidine in high-risk patients with Brugada syndrome. Circulation 2004;110:1731-37. [PubMed: 15381640]

7. Glatter K, Wang Q, Keating M, et al. Effectiveness of sotalol treatment in symptomatic Brugada syndrome. Am J Cardiol 2004;93:1320-22. [PubMed: 15135718]

8. Priori SG, Napolitano C, Gasparini M, et al. Clinical and genetic heterogeneity of right bundle branch block and ST-segment elevation syndrome: a prospective evaluation of 52 families. Circulation 2000;102:2509-15. [PubMed: 11076825]

9. Brugada P, Brugada J. Right bundle branch bock, persistent ST segment elevation and sudden cardiac death: a distinct clinical and electrocardiographic syndrome: a multicenter report. J Am Coll Cardiol 1992;20:1391-96. [PubMed: 1309182]

10. Prior SG, Napolitano C, Gasparini M, et al. Natural history of Brugada syndrome. Insights for risk stratification and management. Circulation 2002;105:1342-47. [PubMed: 11901046] 
11. Antzelevitch C, Brugada P, Borggrege M, et al. Brugada syndrome: report of the Second Consensus Conference. Circulation 2005;111:659-70. [PubMed: 15655131] 


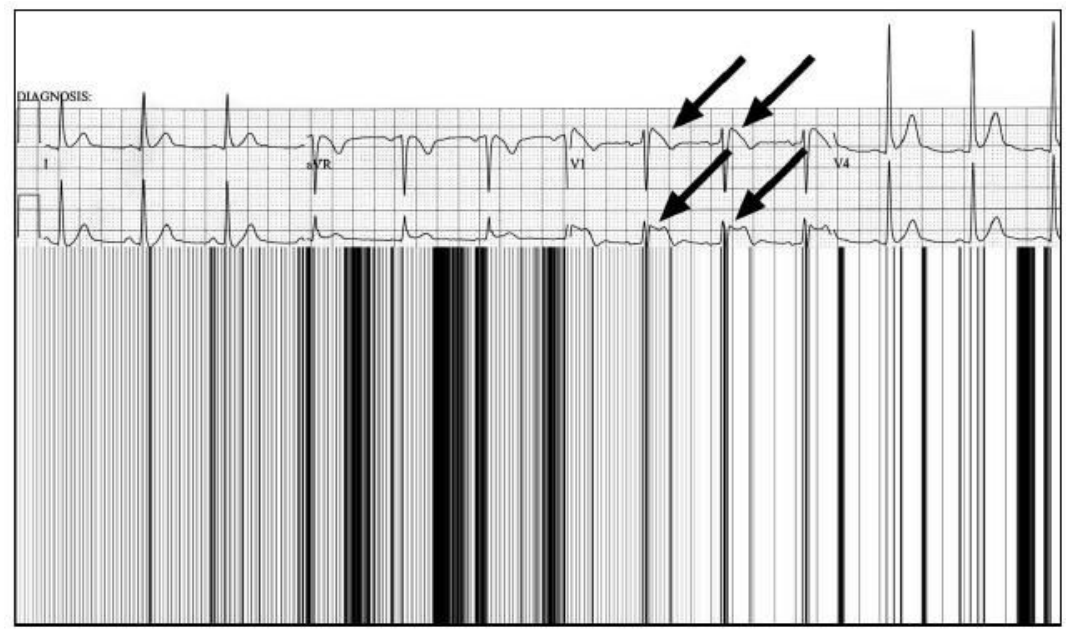

Figure.

12-lead ECG, 46-year old man with family history of sudden death in men. Asymptomatic patient was originally admitted for stab wound to neck and was found to have this ECG classic for Brugada syndrome. Note pseudoright bundle-branch-block pattern and ST-elevation in leads V1-V3 (arrows). Electrophysiology study easily induced ventricular fibrillation, and implantable defibrillator was placed. 